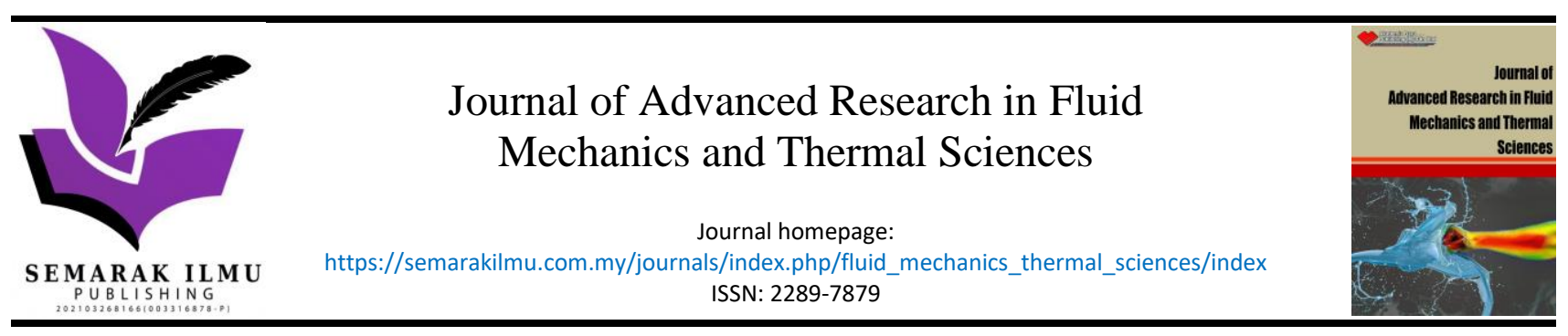

\title{
Numerical Study on Aerodynamic Performance of S809 Wind Turbine
}

\author{
Khurshid Alam ${ }^{1,}{ }^{*}$, Muhammad Saeed ${ }^{2}$, Muhammad Iqbal ${ }^{3}$, Afzal Husain ${ }^{1}$, Himayat Ullah ${ }^{4}$ \\ Department of Mechanical and Industrial Engineering, Sultan Qaboos University, Postal Code 123, Al-Khoud, Sultanate of Oman \\ Mechanical Engineering Department, Khalifa University, PO Box 2533, Abu Dhabi, United Arab Emirates \\ Creative Engineering \& Management Services, Deans Centre Peshawar, Pakistan \\ Centres of Excellence in Applied Sciences and Technologies (CESAT), Islamabad, Pakistan
}

\section{ARTICLE INFO}

\section{Article history:}

Received 28 July 2021

Received in revised form 9 November 2021

Accepted 12 November 2021

Available online 19 December 2021

\section{Keywords:}

Wind turbine; fluid-structure interaction; pressure coefficient; incompressible flow

\section{ABSTRACT}

Wind energy has emerged as one of the cleanest and sustainable sources of energy and is a potential resource for meeting the future's electricity demand. Evaluating the aerodynamic performance of the turbine blade in complex environmental conditions is vital for designing and developing energy-efficient wind turbines. This work aims to undertake aerodynamic analysis of a Horizontal Axis Wind Turbine i.e. NREL Phase IV. Computational fluid dynamics (CFD) models are presented using ANSYS-CFX software. Blade geometries were tested at different wind speeds ranging from $5 \mathrm{~m} / \mathrm{s}$ to $30 \mathrm{~m} / \mathrm{s}$. The power output and pressure coefficients obtained from numerical simulations are compared with experimental data published on wind turbine.

\section{Introduction}

Wind energy is one of the cheapest and effective sources of renewable energy and available in abundance. Electrical energy can be obtained from the wind with the help of wind turbine at suitable locations. Currently, two types of wind turbines exist in the global business frame of renewable energy. i.e. vertical axis wind turbines (VAWT) and horizontal axis wind turbines (HAWT). HAWT is used for commercial applications while VAWT has potential for residential applications. The amount of power a HAWT can produce is determined by the diameter of its rotor. Modern wind turbine blades used for commercial applications have large structures with complex aerodynamic profile of the blade and fabricated from composite materials in sandwich configurations.

The unique structure and material of the turbine blade complicate the design and testing of the wind turbine. Similar to other aerodynamics machines, wind turbines require careful design and analysis for maximum power output at a low cost. As wind turbines having larger blades poses greater challenges in manufacturing and maintenance. Recent advancement in the area is largely focused on efficient structural and aerodynamic designs and the examination of economical and durable materials. Future designs of wind turbine blades will be based on lightweight and high strength

\footnotetext{
* Corresponding author.

E-mail address: kalam@squ.edu.om

https://doi.org/10.37934/arfmts.90.1.154162
} 
materials that can withstand extreme wind conditions. A comprehensive review on the testing of wind turbine blades can be found in recent research studies [1,2].

Much of the research is devoted to improve the efficiency of the wind turbines. The majority of those studies are focused on the aerodynamic performance of a wind turbine blade. Several schemes have been used to evaluate the aerodynamic performance of the wind turbine blade. These include wind tunnel tests, analytical modeling, and numerical simulations. Some recent studies have used advanced features available in the commercially available Finite Element (FE) codes for coupling CFD and FE analysis of wind and tidal turbines [3-8]. Bhadake and Gore [9] presented a detailed review on the CFD analysis of horizontal axis wind turbine blades. They detailed the effect of general aerofoil behaviour and the optimization of aerofoil in order to achieve improved performance. Aerodynamic analysis of wind turbine blade is also discussed in [10]. The authors used a numerical tool called "Aero-T" for the simulation of turbine rotor blades.

Power output from the wind turbine is one of the main objectives and has been studied in several research studies [11,12]. In addition to the power output optimization of the turbine blade for maximum output has also been studied [13-16]. Chord wise surface pressure distributions is one of the important parameter to evaluate performance of wind turbine and has been an interest of several studies $[7,14,17,18]$. Testing wind turbine in real conditions is time-consuming as well as requires expensive testing set up. However, simulating wind turbine through Computational Fluid Dynamics (CFD) software offers inexpensive solutions for efficiently analyzing it. The CFD analysis provide ease in modeling different geometries of turbine blade and wind conditions which helps to optimize the blade geometry with the regard to the power output. Although research work and development activities are currently underway to maximize the power output, not much comparing simulation and experimental data on wind turbine has been published.

In this current work we undertake the aerodynamic performance of an NREL Phase IV Horizontal Axis Wind Turbine. Computational fluid dynamics (CFD) models are presented using ANSYS-CFX software. We consider different blade geometries to test the performance of the blade at different wind speeds ranging from $5 \mathrm{~m} / \mathrm{s}$ to $30 \mathrm{~m} / \mathrm{s}$. The experimental data published in the literature [18] is taken as a reference and the power output and pressure coefficients obtained from numerical simulations of this current study are compared for validation purpose. The results from the simulations show very good agreement with the experimental data.

The remainder of the paper is arranged as follows. The details of the computational model are presented in the proceeding section. The information regarding the geometry of the selected blade and preparation of the computational model using ANSYS-CFX are presented in this section. Section 3 discusses the main numerical results. Some concluding remarks are presented in Section 4.

\section{Computational Model}

\subsection{Geometry}

An NREL Phase IV rotor with 5809 profile and two blades was selected for simulation. The threedimensional model of the NREL Phase VI turbine blade was built using the 2D shape data of S809 aerofoil along with information of twist, chord length and thickness of the profile as a function of the blade span. The 2D aerofile shape data of S809 is given in Table 1 along with the coordinates of the blade in span wise direction in Table 2. The information is also available in reference [3]. Once this information is known geometry of the blade can be modelled by applying this data to generate 28 profiles of the blade at different spans listed in column 1 of Table 2. Each time profile data was picked from Table 1 and was transformed to a new profile by using data in column 2, 3 and 4 of Table 2 . 
Table 1

Aerofile data of S809 profile

\begin{tabular}{|c|c|c|c|}
\hline \multicolumn{2}{|c|}{ Upper Surface } & \multicolumn{2}{|c|}{ Lower Surface } \\
\hline $\mathrm{x} / \mathrm{c}$ & $y / c$ & $x / c$ & $y / c$ \\
\hline 0.00037 & 0.00275 & 0.0014 & -0.00498 \\
\hline 0.00575 & 0.01166 & 0.00933 & -0.01272 \\
\hline 0.01626 & 0.02133 & 0.02321 & -0.02162 \\
\hline 0.03158 & 0.03136 & 0.04223 & -0.03144 \\
\hline 0.05147 & 0.04143 & 0.06579 & -0.04199 \\
\hline 0.07568 & 0.05132 & 0.09325 & -0.05301 \\
\hline 0.1039 & 0.06082 & 0.12397 & -0.06408 \\
\hline 0.1358 & 0.06972 & 0.15752 & -0.07467 \\
\hline 0.17103 & 0.07786 & 0.19362 & -0.08447 \\
\hline 0.2092 & 0.08505 & 0.23175 & -0.09326 \\
\hline 0.24987 & 0.09113 & 0.27129 & -0.1006 \\
\hline 0.29259 & 0.09594 & 0.31188 & -0.10589 \\
\hline 0.33689 & 0.09933 & 0.35328 & -0.10866 \\
\hline 0.38223 & 0.10109 & 0.39541 & -0.10842 \\
\hline 0.42809 & 0.10101 & 0.43832 & -0.10484 \\
\hline 0.47384 & 0.09843 & 0.48234 & -0.09756 \\
\hline 0.52005 & 0.09237 & 0.52837 & -0.08697 \\
\hline 0.56801 & 0.08356 & 0.57663 & -0.07442 \\
\hline 0.61747 & 0.07379 & 0.62649 & -0.06112 \\
\hline 0.66718 & 0.06403 & 0.6771 & -0.04792 \\
\hline 0.71606 & 0.05462 & 0.72752 & -0.03558 \\
\hline 0.76314 & 0.04578 & 0.77668 & -0.02466 \\
\hline 0.80756 & 0.03761 & 0.82348 & -0.01559 \\
\hline 0.84854 & 0.03017 & 0.86677 & -0.00859 \\
\hline 0.88537 & 0.02335 & 0.90545 & -0.0037 \\
\hline 0.91763 & 0.01694 & 0.93852 & -0.00075 \\
\hline 0.94523 & 0.01101 & 0.96509 & 0.00054 \\
\hline 0.96799 & 0.006 & 0.98446 & 0.00065 \\
\hline 0.98528 & 0.00245 & 0.99612 & 0.00024 \\
\hline 0.99623 & 0.00054 & 1 & 0 \\
\hline 1 & 0 & 0 & 0 \\
\hline
\end{tabular}

Operations involved were twisting, scaling $x$-coordinates to get given chord length and scaling in $\mathrm{y}$ direction to get desired thickness at selected span station and stored for that span station. After 28 such iterations profiles of $\mathrm{S} 809$ blade was available to convert this point data to surface data. An inhouse MATLAB code was used for the procedure explained above to get 28 profiles of the blade at different spans. Finally, point data was processed using PTC Creo 2.0 to generate the surfaces of the bade. The final model is shown in Figure 1.

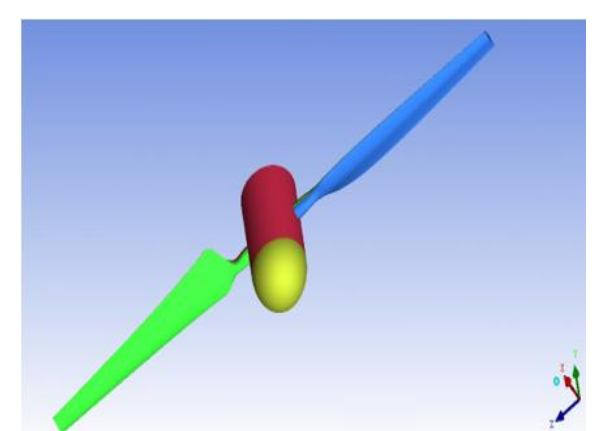

Fig. 1. Two-rotor geometrical model of NREL S809 wind turbine 
Table 2

Span-wise coordinates of the blade

\begin{tabular}{llll}
\hline Distance $r(m)$ & Chord length $(m)$ & Twist (degrees) & Thickness $(\mathrm{m})$ \\
\hline 0 & Hub center & Hub center & Hub center \\
0.5083 & 0.218 & 0 & 0.218 \\
0.6604 & 0.218 & 0 & 0.218 \\
0.8835 & 0.183 & 0 & 0.183 \\
1.0085 & 0.349 & 6.7 & 0.163 \\
1.0675 & 0.441 & 9.9 & 0.154 \\
1.1335 & 0.544 & 13.4 & 0.154 \\
1.2575 & 0.737 & 20.04 & 0.154 \\
1.343 & 0.728 & 18.074 & $20.95 \%$ chord \\
1.51 & 0.711 & 14.292 & $20.95 \%$ chord \\
1.648 & 0.697 & 11.909 & $20.95 \%$ chord \\
1.952 & 0.666 & 7.979 & $20.95 \%$ chord \\
2.257 & 0.636 & 5.308 & $20.95 \%$ chord \\
2.343 & 0.627 & 4.715 & $20.95 \%$ chord \\
2.562 & 0.605 & 3.425 & $20.95 \%$ chord \\
2.867 & 0.574 & 2.083 & $20.95 \%$ chord \\
3.172 & 0.543 & 1.15 & $20.95 \%$ chord \\
3.185 & 0.542 & 1.115 & $20.95 \%$ chord \\
3.476 & 0.512 & 0.494 & $20.95 \%$ chord \\
3.781 & 0.482 & -0.015 & $20.95 \%$ chord \\
4.023 & 0.457 & -0.381 & $20.95 \%$ chord \\
4.086 & 0.451 & -0.475 & $20.95 \%$ chord \\
4.391 & 0.42 & -0.92 & $20.95 \%$ chord \\
4.696 & 0.389 & -1.352 & $20.95 \%$ chord \\
4.78 & 0.381 & -1.469 & $20.95 \%$ chord \\
5 & 0.358 & -1.775 & $20.95 \%$ chord \\
5.305 & 0.328 & -2.191 & $20.95 \%$ chord \\
5.532 & 0.305 & -2.5 & $20.95 \%$ chord
\end{tabular}

\subsection{Model and Meshing}

Meshes for both rotating (inner domain with rotor) and outer stationary domains were generated using ANSYS ICEM CFD. For the rotating domain, the mesh consists of tetrahedral elements with 10 layers of prism elements in the boundary layer region to maintain the required values of $\mathrm{Y}^{+}$i.e $<2$. The computational mesh contains 2.7 million prismatic cells. In order to minimize the errors introduced by the mesh size, a preliminary study was performed with different mesh densities to obtain a converged solution. The results presented in this article are produced with the optimized mesh. Mesh domain around blades is shown in Figure 2. On the other hand, mesh for the stationary domain was generated using hexahedral elements. The size of the outer domain was optimized to avoid pressure gradients at all its boundaries. The computational mesh contains 2.7 million cells.

Continuity, momentum, and energy equations were solved using a commercial code ANSYS-CFX. A large number of turbulence models are available in CFX, some have very specific applications while others can be applied to a wider class of flows with a reasonable degree of confidence. To compute the unknowns in Reynold stress tensor, shear stress turbulence (SST) model was adopted. The SST model is based on the k- $\omega$ model and accounts for the transport of the turbulent shear stress and gives accurate predictions of the onset and the amount of flow separation. The SST turbulence model provides a good compromise between the accuracy and computational expense.

Inlet of the stationary domain was assigned with velocity boundary condition while pressure conditions were imposed at its outlet. The inner domain was assigned with a rotational speed of 72 
$\mathrm{rpm}$. The interfaces between the rotating and stationary domains were solved using the frozen rotor model. An air was considered as fluid flowing against the turbine blades. The governing equations were Reynolds averaged Navier-Stokes equations for three dimensional, viscous, incompressible flow and continuous equation. The boundary conditions were implemented by selecting a velocity inlet upwind from the blades. At the inlet, turbulence intensity was fixed to $10 \%$. An outflow condition is specified downwind.

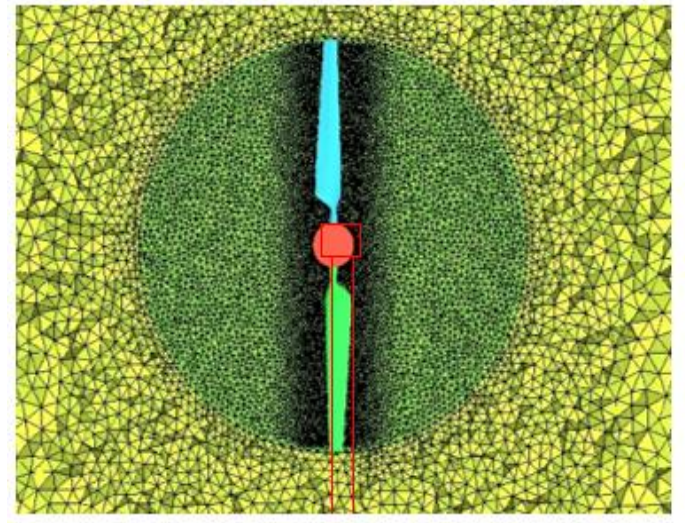

(a)

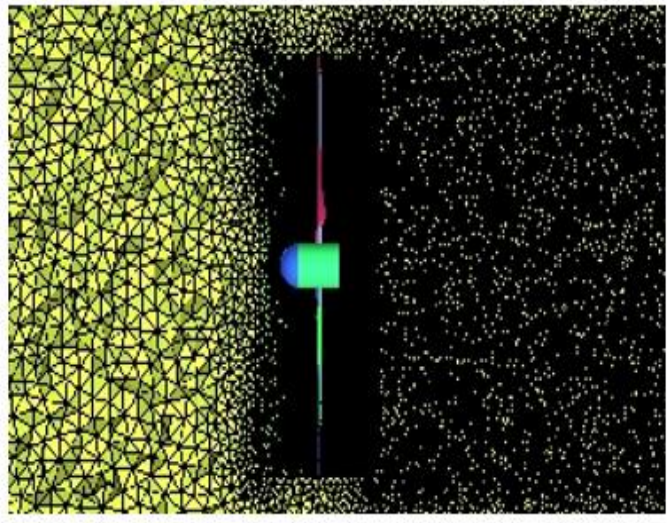

(b)

Fig. 2. Meshed domain around blades (a) front side (b) side view

\section{Results}

The pressure distribution on the rotating blade surface depends on the inflow velocity, angle of attack, and the profile of the surface of the blade. Non-dimensional parameters were adopted while performing data reduction procedures. Figure 3 shows the pressure contours of the pressure plane and suction plane of the blade. Figure 3 shows the pressure contours of the pressure plane and suction plane of the blade. Figure 4 shows velocity contours around the blade.

Three different levels of wind speeds $(5 \mathrm{~m} / \mathrm{s}$ to $30 \mathrm{~m} / \mathrm{s}$ ) were considered to find the effect of wind velocity on the power output. The relationship between wind speed and power output is shown in Figure 5 . The power output was found to be strongly affected by the wind speed as revealed by experiments and CFD analysis. The power generation section of the blade was from $60 \%$ diameter to the tip of the blade. The experimental data published in the literature [19] is used for comparison with numerical results in the subsequent plots. The results from the simulations show very good agreement with the experimental data.

The amount of lift on the blade strongly depends on the difference between the pressure on the upper surface (suction surface) and the lower surface (pressure surface). A high negative pressure occurs on the leading edge of the suction surface and is gradually increased along the trailing edge. The pressure distribution integrated around the blade section provides a sectional lift coefficient. A non-dimensional pressure coefficient $C_{p}$ was considered at two span-wise sections (i.e. $30 \%$ and $80 \%$ ). The data presented in the plots were obtained at a constant wind speed of $10 \mathrm{~m} / \mathrm{s}$ and $15 \mathrm{~m} / \mathrm{s}$.

Figure 6 shows comparison of pressure coefficient between field experiments and CFD results corresponding to aerofoil profiles S809. A greater differential was found at the leading edge than the trailing edge along the span closer to the root. More differential pressure was found in the experiment compared to numerical findings away from the root of the blade. Figure 7 shows more pressure differential between pressure plane and the suction plane of the blade at span of $80 \%$. This may be attributed to the rotation effect and flow separation on the blade away from the root of the blade. Simulations predicted more differential pressure compared to experimental results at span 
$30 \%$ (closed to the root). More consistency between experimental results and numerical results was found away from the root of the blade. Separation of the vortex at the trailing edge had more influence on the pressure distribution on the surface of the blade.

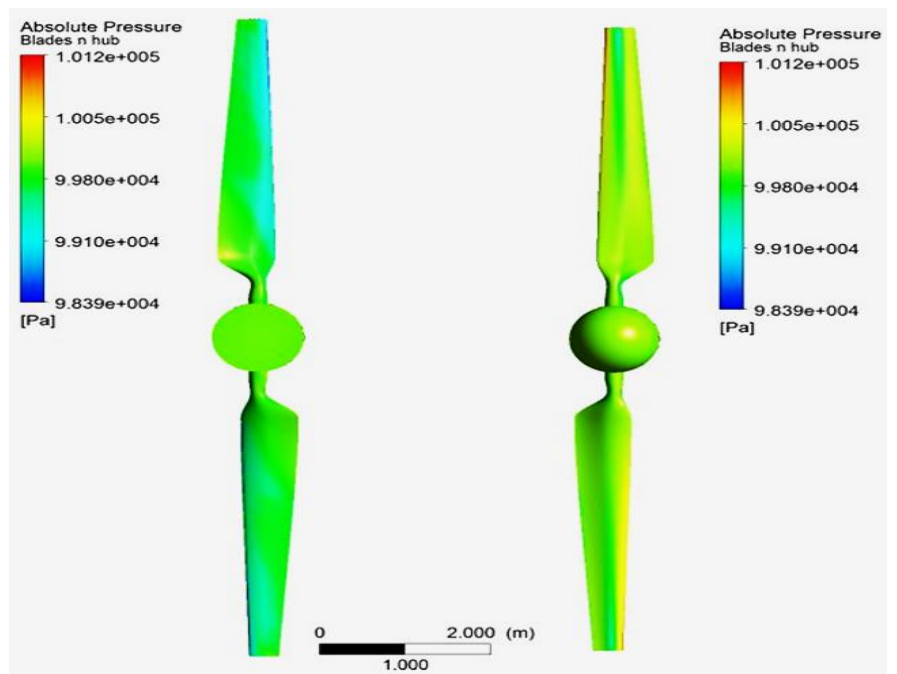

Fig. 3. Pressure distribution on blades. Left - back side of the blade, right - front side of the blade

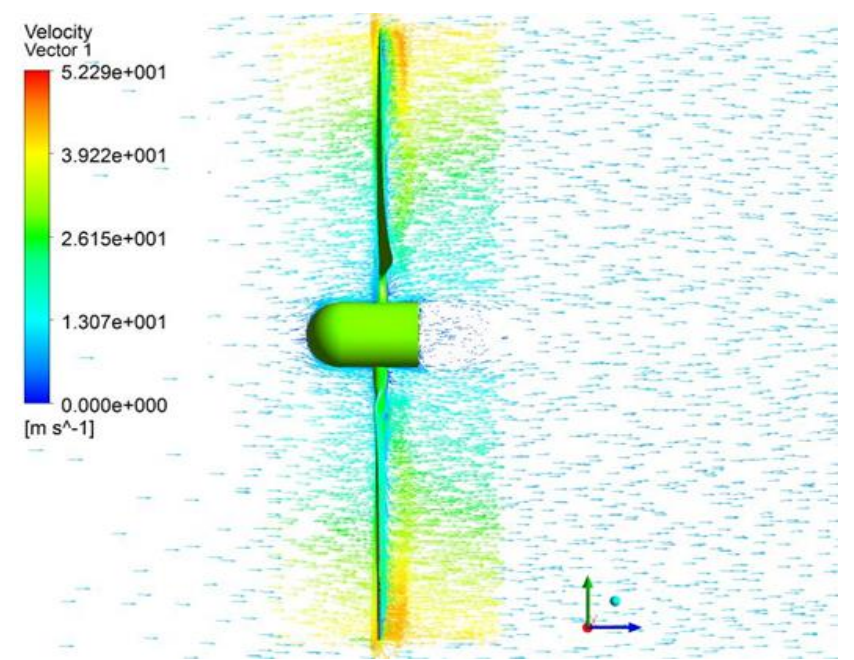

Fig. 4. Velocity distribution on blades

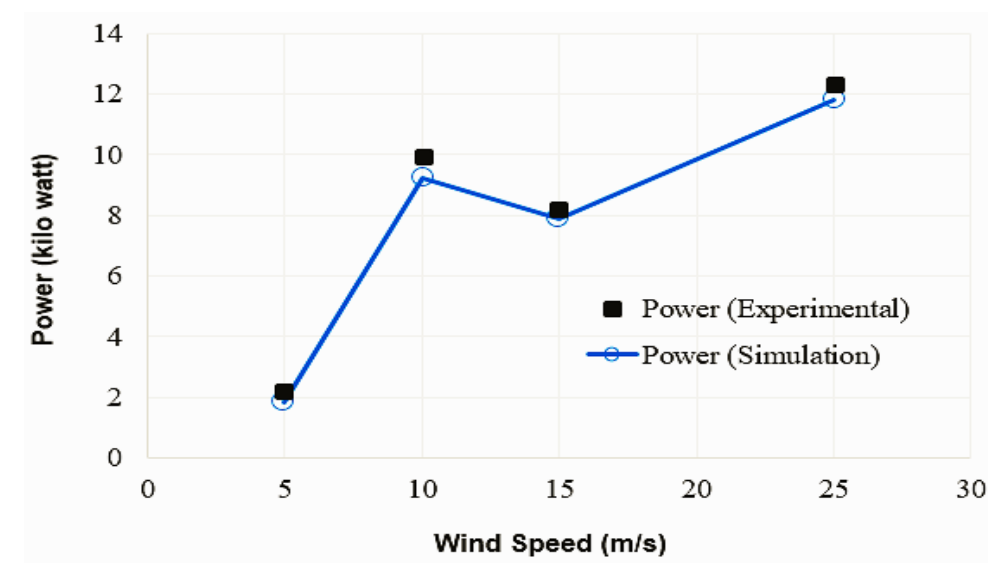

Fig. 5. Comparison of power between experimental and numerical results 


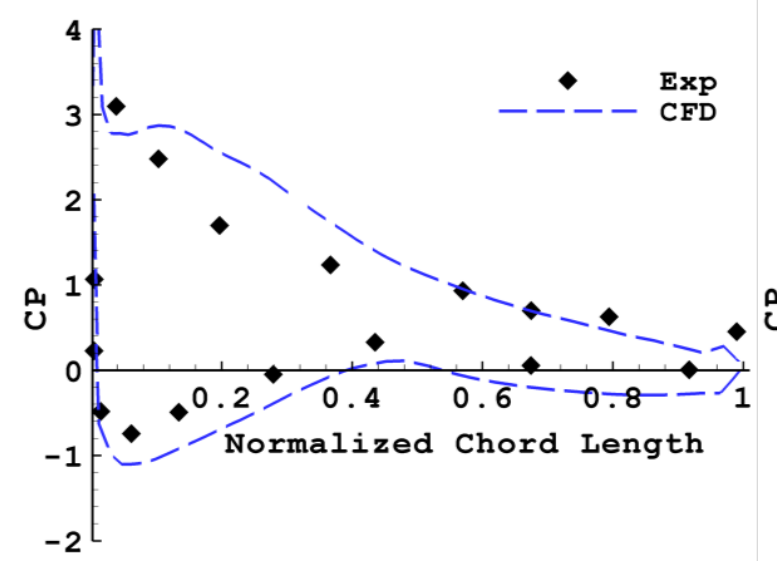

(a)

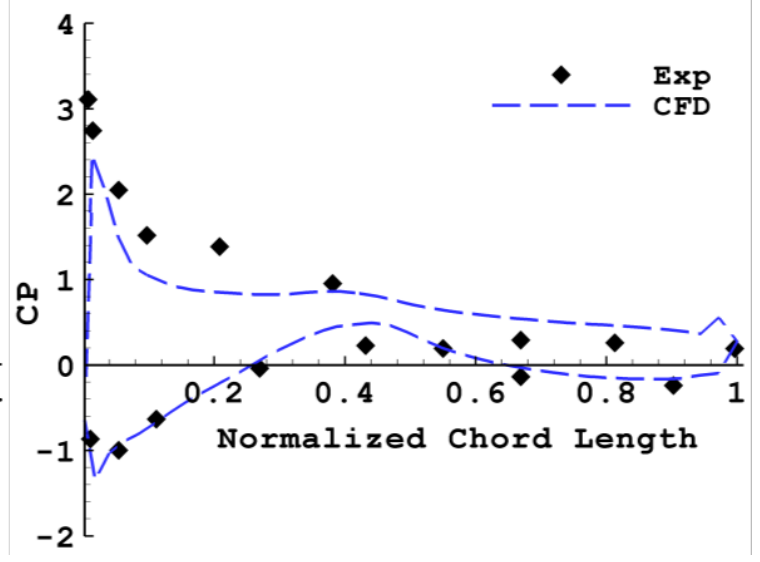

(b)

Fig. 6. Comparison of pressure coefficient, (a) span $=30 \%$, (b) span $=80 \%$, wind speed $10 \mathrm{~m} / \mathrm{s}$

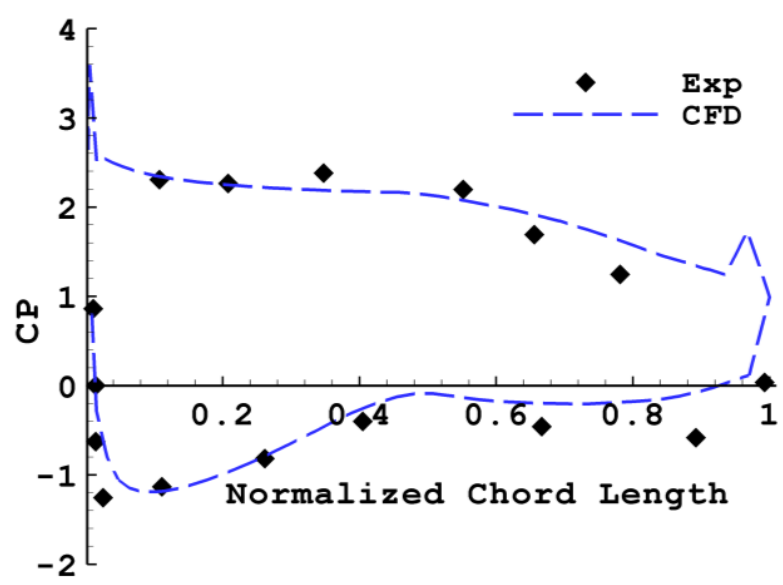

(a)

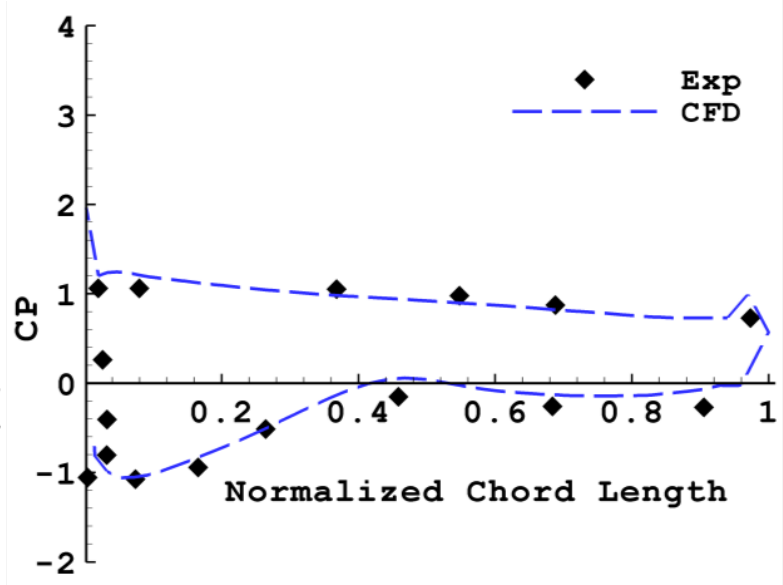

(b)

Fig. 7. Comparison of pressure coefficient, (a) span $=30 \%$, (b) span $=80 \%$, wind speed $15 \mathrm{~m} / \mathrm{s}$

Closed match between experimental and numerical results revealed CFD a powerful tool for modelling complex interaction between air and turbine blades. However, modelling a complex interaction of volatile air (storm, air with hails and humidity etc.) with composite turbine blade is a challenging task. Modelling interaction between air as a multiphase flow and turbine blade with multiple composite layers could be a more realistic and demanding for the research community.

\section{Conclusion}

In the present study, an attempt has been made to perform aerodynamic performance of NREL S809 blade using CFD analysis and compare the results with published experimental data on the prescribed turbine blade. In the chosen range of wind speed, the power output was almost increased linearly with increase in wind speed. Similarly, the pressure coefficient obtained from experiments and CFD analysis was found in good agreement for all values of wind speed. Closed agreement between experimental and simulation results showed that CFD analysis can be confidently carried out to evaluate aerodynamic performance of the blade profiles of wind turbines of different geometries under volatile wind conditions. 


\section{Acknowledgements}

This research study is sponsored by The Research Council (TRC) of Sultanate of Oman (Grant No: RC/RG-ENG/MIED/19/01).

\section{References}

[1] Hossain, Md Liton, Ahmed Abu-Siada, and S. M. Muyeen. "Methods for advanced wind turbine condition monitoring and early diagnosis: A literature review." Energies 11, no. 5 (2018): 1309. https://doi.org/10.3390/en11051309

[2] Yang, Bin, and Dongbai Sun. "Testing, inspecting and monitoring technologies for wind turbine blades: A survey." Renewable and Sustainable Energy Reviews $22 \quad$ (2013): 515-526. https://doi.org/10.1016/i.rser.2012.12.056

[3] Shkara, Yasir, Martin Cardaun, Ralf Schelenz, and Georg Jacobs. "Aeroelastic response of a multi-megawatt upwind horizontal axis wind turbine (HAWT) based on fluid-structure interaction simulation." Wind Energy Science 5, no. 1 (2020): 141-154. https://doi.org/10.5194/wes-5-141-2020

[4] Zhu, Rui, Da-duo Chen, and Shi-wei Wu. "Unsteady flow and vibration analysis of the horizontal-axis wind turbine blade under the fluid-structure interaction." Shock and Vibration 2019 (2019). https://doi.org/10.1155/2019/3050694

[5] Borouji, Ehsan, and Takafumi Nishino. "Fluid Structure Interaction Simulations of the NREL 5 MW Wind TurbinePart I: Aerodynamics and Blockage Effect." Journal of Offshore Mechanics and Arctic Engineering 141, no. 2 (2019). https://doi.org/10.1115/1.4040980

[6] Halawa, Amr M., Basman Elhadidi, and Shigeo Yoshida. "Aerodynamic performance enhancement using active flow control on DU96-W-180 wind turbine airfoil." Evergreen 5, no. 1 (2018): 16-24. https://doi.org/10.5109/1929723

[7] Wang, Lin, Robin Quant, and Athanasios Kolios. "Fluid structure interaction modelling of horizontal-axis wind turbine blades based on CFD and FEA." Journal of Wind Engineering and Industrial Aerodynamics 158 (2016): 1125. https://doi.org/10.1016/i.jweia.2016.09.006

[8] Badshah, Mujahid, Saeed Badshah, James VanZwieten, Sakhi Jan, Muhammad Amir, and Suheel Abdullah Malik. "Coupled fluid-structure interaction modelling of loads variation and fatigue life of a full-scale tidal turbine under the effect of velocity profile." Energies 12, no. 11 (2019): 2217. https://doi.org/10.3390/en12112217

[9] Bhadake, Ms Priti G., and V. G. Gore. "A Review on Aerodynamic Analysis of Horizontal Axis Wind Turbine Blade Using CFD Technique." International Journal of Engineering and Applied Sciences 3, no. 4: 257691.

[10] Martinez, J. A. V. I. E. R., Piotr Doerffer, Oskar Szulc, and Fernando Tejero. "Aerodynamic analysis of wind turbine rotor blades." Task Q 19, no. 2 (2015): 129-140.

[11] Zhu, Jie, Zhong Zhou, and Xin Cai. "Multi-objective aerodynamic and structural integrated optimization design of wind turbines at the system level through a coupled blade-tower model." Renewable Energy 150 (2020): 523-537. https://doi.org/10.1016/j.renene.2020.01.013

[12] Li, D. S., R. N. Li, L. J. Wei, X. Y. Wang, Y. Qiang, and Y. R. Li. "Comparison of the pressure distribution of a wind turbine blade based on field experiment and CFD." In IOP Conference Series: Materials Science and Engineering, vol. 52, no. 5, p. 052004. IOP Publishing, 2013. https://doi.org/10.1088/1757-899X/52/5/052004

[13] Zhu, Jie, Xiaohui Ni, and Xiaomei Shen. "Aerodynamic and structural optimization of wind turbine blade with static aeroelastic effects." International Journal of Low-Carbon Technologies 15, no. 1 (2020): 55-64. https://doi.org/10.1093/ijlct/ctz057

[14] Lee, Hak Min, and Oh Joon Kwon. "Performance improvement of horizontal axis wind turbines by aerodynamic shape optimization including aeroealstic deformation." Renewable Energy 147 (2020): 2128-2140. https://doi.org/10.1016/i.renene.2019.09.125

[15] Polat, Ozge, and Ismail H. Tuncer. "Aerodynamic shape optimization of wind turbine blades using a parallel genetic algorithm." Procedia Engineering 61 (2013): 28-31. https://doi.org/10.1016/i.proeng.2013.07.088

[16] Jureczko, M. E. Z. Y. K., M. Pawlak, and A. Mężyk. "Optimisation of wind turbine blades." Journal of materials processing technology 167, no. 2-3 (2005): 463-471. https://doi.org/10.1016/i.jmatprotec.2005.06.055

[17] Phengpom, Tinnapob, Yasunari Kamada, Takao Maeda, Tasuku Matsuno, and Noriaki Sugimoto. "Analysis of wind turbine pressure distribution and 3D flows visualization on rotating condition." IOSR Journal of Engineering 6, no. 02 (2016): 18-30.

[18] Mazlan, Mohamad Zahid, Fazila Mohd Zawawi, Teeab Tahzib, Kamarulafizam Ismail, and Syahrullail Samion. "Performance Analysis of Highway Wind Turbine Enhanced with Wind Guide Vanes Using the Taguchi Method." CFD Letters 13, no. 3 (2021): 25-42. https://doi.org/10.37934/cfdl.13.3.2542 
[19] Simms, David, Scott Schreck, Maureen Hand, and Lee Jay Fingersh. NREL unsteady aerodynamics experiment in the NASA-Ames wind tunnel: a comparison of predictions to measurements. No. NREL/TP-500-29494. National Renewable Energy Lab., Golden, CO (US), 2001. https://doi.org/10.2172/783409 